\title{
Parametric Study on Formation Flying Effectiveness for a Blended-Wing UAV
}

\author{
Hyo-Sang Shin ${ }^{1}$ (D) . Antonis F. Antoniadis ${ }^{1}$ - Antonios Tsourdos ${ }^{1}$
}

Received: 28 September 2017 / Accepted: 10 April 2018 / Published online: 26 April 2018

(C) The Author(s) 2018

\begin{abstract}
This paper investigates aerodynamic performance improvements of formation flight at transonic speeds for a medium size Unmanned Aerial Vehicle (UAV). The metric for assessing the aerodynamic improvement of formation flight is the computed drag. The total drag for each formation configuration is compared with a single UAV, where a final drag reduction percentage is estimated. The evaluation of the aerodynamic performance is conducted by employing an in-house Computational Fluid Dynamics (CFD) solver, grid generation and post processing tools. For critical understanding of the tendency of the formation efficiency depending on main parameters, broad formation configurations are analysed. The parameterisation includes number of aircraft, proximity and formation shape. Full realisation of the benefit predicted would need to be proven in the real world, but there is sufficient confidence to suggest that it exist: the empirical parametric analysis suggests that formation flight can improves aerodynamic performance and formation configuration greatly influence the degree of improvement.
\end{abstract}

Keywords Formation flying $\cdot$ CFD analysis $\cdot$ Aerodynamic improvement $\cdot$ Drag reduction percentage $\cdot$ Formation configuration matrix

\section{Introduction}

Formation flight has been a topic of research across various scientific sectors. Biologists have studied the energy savings of large birds flying in flocks usually in vtype formation. Research was carried out demonstrating the aerodynamic efficiency improvements of birds in formation flight both with respect to flight measurements and within a simulation framework [1,2]. It is widely believed that formation flying of aircraft could bring tactical advantages in operating multiple aircraft and could improve the aerodynamic efficiency. To validate the hypothesis on the aerodynamic performance improvements from the formation flying in aircraft operation, aerodynamicists have performed numerous studies.

Hyo-Sang Shin

h.shin@cranfield.ac.uk

Antonis F. Antoniadis

a.f.antoniadis@cranfield.ac.uk

Antonios Tsourdos

a.tsourdos@cranfield.ac.uk

1 SATM, Cranfield University, Cranfield MK43 0AL, UK
The evaluation is generally conducted by measuring and computing the flow physics and forces exerted on aircraft, and by computing the aerodynamic improvements of formation flight. For instance, a comprehensive study has been performed by NASA [3] where two F/A-18 aircraft flying in tight formation reached a $20 \%$ drag reduction and $18 \%$ fuel savings. The difference between tight and extended flight formations lies with the streamwise proximity, usually an extended formation is greater than 10 wing-spans, a tight formation is much smaller.

Recently, there have been several studies published, presenting aerodynamic studies for both tight and extended aircraft formation flight [4-6]. An aerodynamic performance for extended formation is conducted by Ning et al. [6] where 30\% drag reduction is achieved with two aircraft and $40 \%$ with three aircraft. An inviscid CFD simulation was performed by Kless et al. [5], where extended formation flight of two aircraft was studied with drag reduction of 54 and 35\% for subsonic and transonic flows, respectively. A study conducted by Kaden and Luckner [7] employed a discrete vortex filament method to model wake vortex rollup for tight formation flight where it was concluded that the inclusion of fluid physics such as viscosity Reynolds number are required for verification and validation of current 
modelling approaches for formation flight. There have been also recent studies undertaken to find an optimum spacing between the various units in formation and some studies cased light upon the effect of shape and size of the leader and follower $[8,9]$.

Most of these studies, if not all, considered only a small number of aircraft in formation, generally one to three aircraft. Considering the complexity of the analysis and typical number of manned aircraft in formation for military and civilian applications, the investigation based on a small number of aircraft could be justifiable. This might become different, when considering formation flight of Unmanned Aerial Vehicles (UAVs). There have been many studies, which investigated and demonstrated strategic and tactical advantages of having many number of UAVs in formation flying. Moreover, there have been increasing attentions on the possibility of operating UAV swarms, where the number of UAVs has different orders of magnitude to formation. Nevertheless, to the best of the author's knowledge, there are no studies investigating aerodynamic performance improvements of formation flying with a increased number of UAVs.

This paper examines aerodynamic performance improvement in UAV formation flying. As UAV formation could consist of increased numbers of aircraft, configurations of formation could become critical. In our previous study [10], we performed an initial investigation on several configurations of UAV formation and carried out initial analysis on the effect of those configurations on the aerodynamic performance. Note that in this study, the number of UAVs was limited to seven aircraft and only two types of formation configurations were considered. The initial investigation revealed that the formation configuration might have a significant impact on the aerodynamic performance of the formation. To this end, this paper aims to extend our previous investigation and further analyse the effect of formation configurations on the aerodynamic performance. The investigation considers the number of aircraft in formation up to ten and includes three types of formation configurations.

Formation flight aerodynamic benefits are mainly attributed to induced-drag reduction, which is present due to the wake vortices generated from the leader aircraft's wing tip. The pressure difference between the upper and lower wing drives the flow to roll-up at the wing tips. The following aircraft tries to find the "sweet spot" making use of the leader's wing-tip vortices utilising the pressure gradient to increase his lift.

From a CFD perspective, the most accurate simulation for capturing wake vortex physics is Direct Numerical Simulations (DNS) followed by Large Eddy Simulations (LES) which both are prohibited due to their immense computational cost; Reynolds Average Navier-Stokes (RANS) is a fair trade-off between the accuracy and cost, capturing efficiently the main features of the these flows. To the best of our knowledge, even the most advanced 3D CFD formation flight simulations in published studies, employ Euler equations with some kind of vortex correction models without turbulence modelling; thus neglecting several physical aspects related to turbulence. However, wake vortex evolution, roll-up and decay has been extensively studied with the employment of sophisticated fluid dynamics models and methods. Hybrid methods i.e. Detached Eddy Simulation (DES), Scale Adaptive Simulation (SAS), hybrid RANSILES are becoming popular as to combine the robustness and speed of RANS models for near body computations and high-resolution methods for the wake vortex formations in the far field [11, 12].

As the main objectives of the current analysis is the modelling of great number of formation types and configurations with as much as ten aircraft, 3D computations even with the Euler's equation (inviscid) would be a tremendous computational effort. For an initial investigation, two-dimensions would be ideal as large number of test-cases can be readily performed in a feasible period of time by exploring several configurations and parameters. However, it will be essential to incorporate the 3D wake vortices and induced drag in the 2D-based investigation. Therefore, this paper applies the RANS that is also applied in our previous study [10]. Note that Reynolds number, viscosity and compressibility effects are taken into account in the RANS. These effects are not considered even in current 3D formation flight simulations and, as discussed, the RNAS provides a fair trade off between the computational cost and accuracy. 2D simulation based on the RANS will provide a good approximation for an initial test phase.

In the analysis, drag reduction percentage is examined for a total of 17 formation configurations. Despite we perform 2D-based investigation, the predicted drag reductions for two and three aircraft are similar with drag reduction estimates of reported studies. The analysis results strongly suggest that configuration parameters, such as number of UAVs in the formation and its shape, significantly influence the degree of the drag reduction: from the analysis, the most efficient formation is the diamond type, followed by the half-diamond then the v-type and final the echelon. Note that, recognising the limitations of the methods used, full realisation of the benefit predicted would need to be proven in the real world. However, the parametric study in this paper provides sufficient confidence to suggest the aerodynamic benefit of formation and its tendency with respect to different configurations.

The paper starts with an overview of the physics involved in transonic aerodynamic flows; the governing equations and turbulence model are detailed. A brief description of the in-house solver with discretisation and numerical schemes is presented followed by the grid generation 
work. The flight envelope and simulation matrix are explained including a preliminary study on the stream-wise proximity. The aerodynamic benefits of each configuration are assessed in the results and discussion section. Finally, the main outcomes are summarised followed by future work directions, recommendations including preliminary results from a 3D simulation.

\section{Framework Modelling}

This section is devoted to the governing equations, numerical methods and grid generation. The in-house solver employed for the current simulation is detailed, demonstrating current and future capabilities related to formation flight physics. Note that the framework modelled in this section is based on the one in our previous study [10].

\subsection{Governing Equations}

The most accurate mathematical expression of fluid dynamics lies with the 3D compressible Navier-Stokes equations, where physical phenomena encountered in formation flight can be accurately predicted i.e. compressibility, boundary layer separation, transition to turbulence, shockwaves, wake formation, evolution and decay.

As the main objectives of the current analysis is the modelling of great number of formation types and configurations with as much as ten aircraft, 3D computations even with the Euler's equation (inviscid) would be a tremendous computational effort. To put it in perspective a 3D grid for inviscid simulation (no prism layer) with local refinement in the wake, to be able to capture the wake-vortex, will be composed of approximately 1.5 to 2 millions elements and this will correspond only two half the aircraft with symmetry boundary conditions imposed on the $(\mathrm{X}, \mathrm{Y})$ plane. A 3D grid was generated for a typical blended-wing stealth unmanned combat aerial vehicle shown in Fig. 1. One simulation with this grid will take from 1 to 3 days in a modern desktop computer running in six CPUs, depending upon the physics models, numerical algorithms convergence criteria etc. This doesn't include the grid generation procedure which can take up to 2 days for a good quality unstructured 3D grid (1 aircraft).

Two-dimensions would be ideal in the initial investigation as large number of test-cases can be performed in a reasonable period of time by exploring several configurations and parameters. The main drawback of $2 \mathrm{D}$ is the
Fig. $13 \mathrm{D}$ grid for a typical blended-wing UAV. The grid was generated and composed of approximatly 2 million tetrahedrals, note that the grid is created for half the geometry, here the aircraft surface is mirrored in the $(\mathrm{X}, \mathrm{Y})$ plane for visualisation purposes

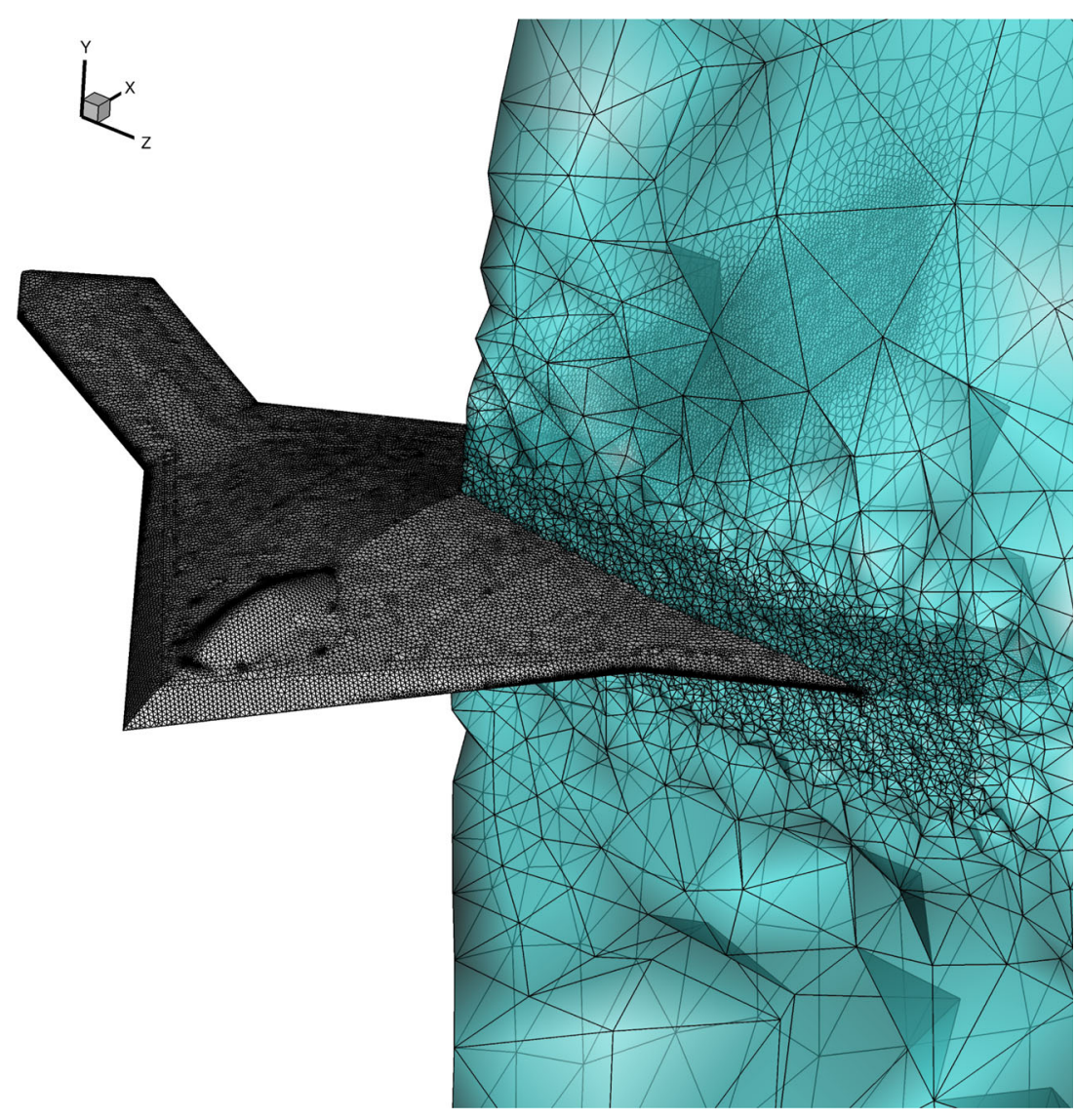


Fig. 2 Residual history for 3 UAVs in V-type formation

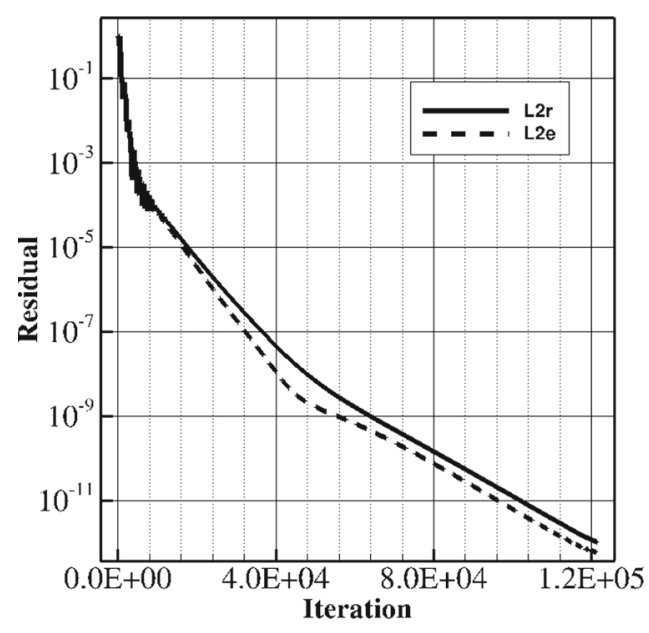

(a) Residual of $\lambda 2$ norm for density and energy

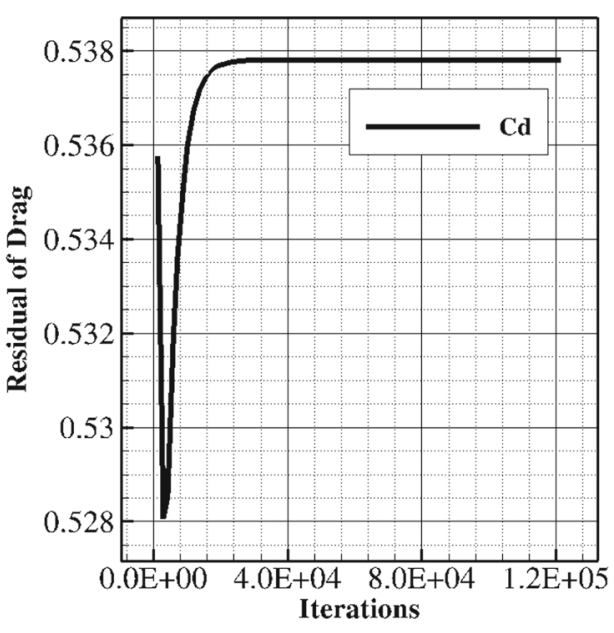

(b) Residual of $C_{d}$ inherited assumption of two-dimensionality where real 3D wake vortices and induced drag effects cannot be modelled. Nevertheless, two-dimensional simulation provides a good approximation for a blind test phase; in addition for the current study the Reynolds Average Navier-Stokes (RANS) are considered where Reynolds number, viscosity and compressibility effects are accounted for; this effect are not considered in current 3D formation flight simulations.

The CFD tool solves the RANS equations which are formulated for the finite volume method and discretised on hybrid unstructured elements: triangles and quadrilaterals. The 2D RANS equations are written in integral form as

$\frac{\partial}{\partial t} \int_{V_{i}} \mathbf{W}_{\mathbf{i}} d V_{i}+\oint_{\partial V_{i}}\left[\left(\mathbf{F}^{\mathrm{c}}-\mathbf{F}^{\mathrm{v}}\right) \mathbf{n}_{\partial V_{i}}\right] d A_{\partial V_{i}}=0$

where $i$ is the index of an element with volume $V_{i}, \mathbf{W}_{\mathbf{i}}$ is the vector of conserved variables, $\mathbf{F}^{\mathrm{c}}$ is the vector of inviscid fluxes and $\mathbf{F}^{\mathrm{v}}$ is the vector of viscous fluxes, the outwardpointing unit normal vector is labelled as $\mathbf{n}=\left(n_{x}, n_{y}\right)$. The equations are dimensionalised according to the free-stream Mach number $M_{\infty}$.

Air is considered as an ideal gas and according to Boussinesq's approximation relating the viscous stress tensor $\tau_{i j}$ to the Reynolds stresses through the eddy viscosity $\mu_{t}$, effectively modelling the momentum transfer by turbulent eddies, written as

$$
\begin{aligned}
\tau_{i j} & =2\left(\mu_{l}+\mu_{t}\right)\left(s_{i j}-\frac{1}{3} \frac{\partial u_{k}}{\partial x_{k}} \delta_{i j}\right), \text { for } i=1,2 j=1,2 \\
s_{i j} & =\frac{1}{2}\left(\frac{\partial u_{i}}{\partial x_{j}}+\frac{\partial u_{j}}{\partial x_{i}}\right)
\end{aligned}
$$

where $k$ is the thermal conductivity, $\delta_{i, j}$ is the Kronecker delta. The eddy viscosity is computed with the SpalartAllmaras (SA) one-equation turbulence model [13]. The model is widely employed for aerospace application as it is robust across subsonic to supersonic regimes. The model solves one transport quantity the turbulence parameter $\tilde{v}$ which is related to eddy viscosity $\mu_{t}$ as

$\mu_{t}=\rho \tilde{v} f_{v 1} \quad$ where $\quad f_{v 1}=\frac{\left(\rho \tilde{v} / \mu_{l}\right)^{3}}{\left(\rho \tilde{v} / \mu_{l}\right)^{3}+C_{v 1}^{3}}$ and $C_{v 1}=7.1$

the SA model equation is written as

$$
\begin{aligned}
\frac{d(\rho \tilde{\nu})}{d t}= & C_{b 1} \tilde{S} \rho \tilde{v}+\frac{1}{\sigma}\left(\nabla \cdot\left(\mu_{l}+\rho \tilde{v}\right) \nabla \tilde{v}\right)+\frac{1}{\sigma} C_{b 2} \rho(\nabla \tilde{v})^{2} \\
& -C_{w 1} f_{w} \rho\left(\frac{\tilde{\nu} k}{d}\right)^{2}
\end{aligned}
$$

where $C_{b 1}, \tilde{S}, \sigma, C_{b 2}, C_{w 1}, f_{w}, \kappa$ are functions and constants of the model and $d$ is the distance from the nearest wall boundary.

\subsection{Solver Description}

The in-house solver is capable of handling hybrid unstructured grids, where the discretisation scheme evaluate its quantity and gradient according to several numerical schemes. For fast simulations a $1^{s t}$ and $2^{n d}$-order MUSCLTVD (Monotone Upstream-centred Schemes for Conservation Laws - Total Variation Diminishing) scheme. Detailed description of the methods, numerical algorithms and applications can be found in published journal papers [14-17].

For the current study a first order spatial discretisation is employed where the gradients of temperature, velocities and turbulence viscosity are approximated with the GreenGauss method. The Rusanov Riemann solver [18] handles the computation of the inviscid intercell fluxes as it is very stable for blunt body aerodynamics at transonic speeds. The implicit LU-SGS time discretisation scheme is employed to advance the time to a steady state [19]. Convergence 
Fig. 3 Geometry and configuration of the BAE-systems Corax/Raven UCAV (http://www.unicraft.biz/ on/corax/corax.htm)

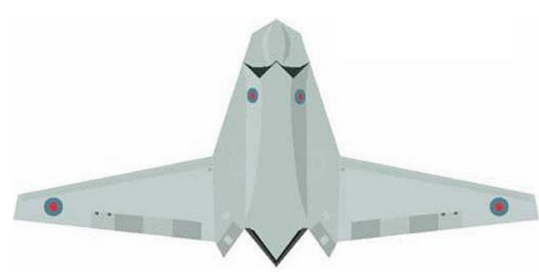

(a) Top view

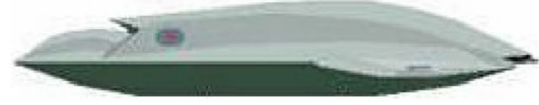

(b) Side view is monitored with the residual of the mean equations and turbulence quantities as well as with the computed drag. Typical convergence behaviour can be depicted from Fig. 2 where the residual histories for 3 UAVs in V-type formation are shown for the density, energy and drag. The solution is assumed to be converged where the drag is stabilised around forty thousand iterations.

\subsection{Geometry and Grid Generation}

As acknowledged, this study is supported by BAE Systems (Operations). The UAV geometry is based on an approximate design of the BAE-systems blended wing UAV "Corax/Raven" shown in Fig. 3. The wing-span is assumed to be around 10 metres which is used for the estimation of the Reynolds number. Both top and side view 2D simulation with one aircraft are performed, however the side view approach prove to be misleading as the lift remained very small as the simulated 2D plane was set on half way of the wing-span. In addition the side view would limit the number of possible combinations of formation configuration, therefore the top view was selected to perform the CFD analysis. Fig. 4 illustrates the grid for one aircraft with focus both on the body and farfield. Figures 5, 6 and 7 show the grids generated for the echelon, v-type, diamond and half diamond shape formation configurations, respectively.

Once the geometry (points and curves) are extracted from the figures, they are imported in a grid generation software.
Hybrid unstructured grids provide exceptional easy for generation and manipulation of the grid which is ideal for the current analysis. Quadrilateral elements are extruded from the surface and triangular elements are generated for the far-field.

\section{Simulation Set-Up}

This section entails the free-stream conditions, simulation framework, and complete test-case matrix. An initial study is conducted to determine the stream-wise optimal proximity simulating two and three aircraft, once the optimal distance was found it was used for the rest of the test-cases. The aerodynamic efficiency improvement metric is the drag and the results are assessed based on the percentage of drag reduction. The drag reduction is computed based on the average of the drag from the aircraft in formation.

\subsection{Free-Stream Conditions}

A free-stream Mach number of $M_{\infty}=0.7$ is set as freestream condition corresponding to a transonic regime with a corresponding Reynolds number of $R e \approx 75 \times 10^{6}$ based on half of the wing-span. Far-field boundary conditions are imposed on the outer boundaries where flow quantities are computed based on the characteristic speed (local speed of sound).
Fig. 4 Hybrid unstructured 2D grid for one UAV configuration composed of 29,962 elements

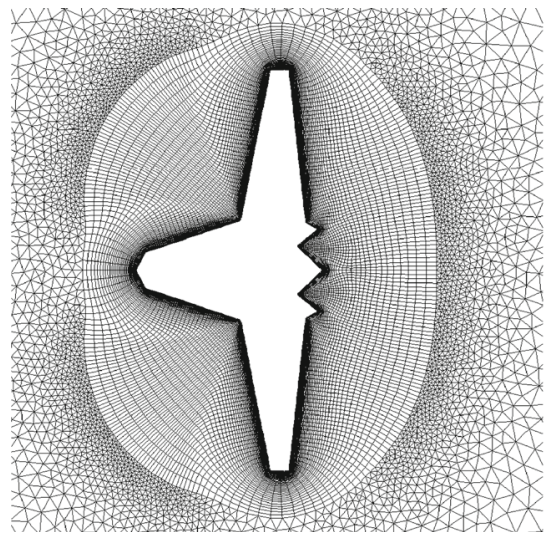

(a) Grid focused on the UCAV

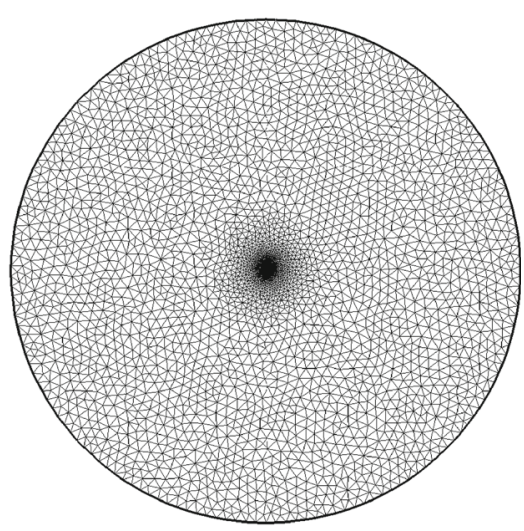

(b) Far-field Grid 


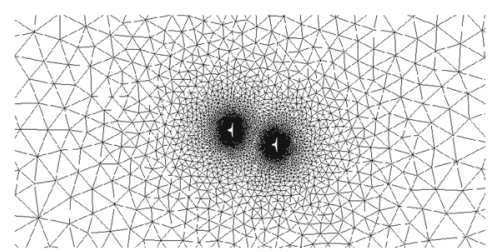

(a) Echelon 2

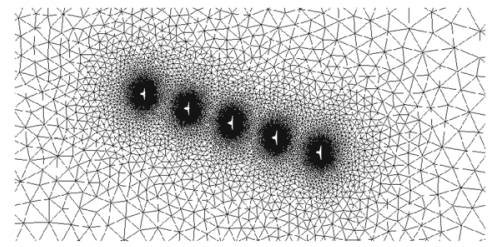

(d) Echelon 5

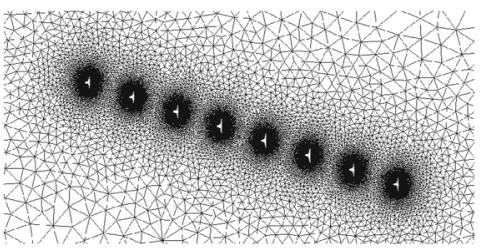

(g) Echelon 8

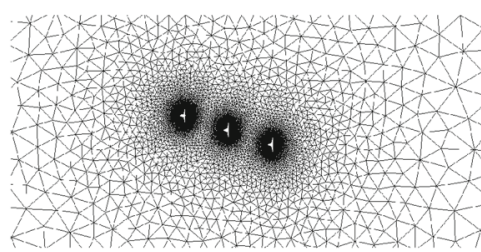

(b) Echelon 3

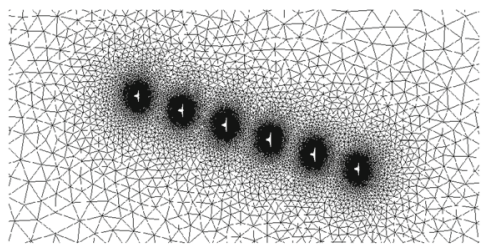

(e) Echelon 6

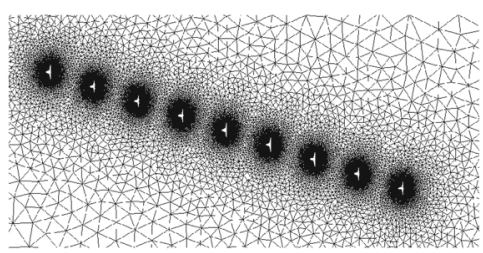

(h) Echelon 9

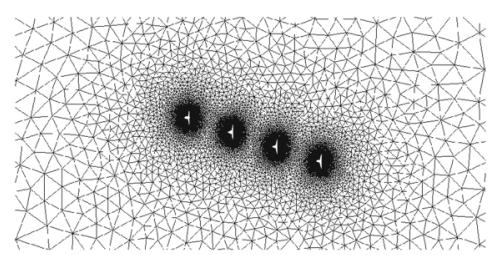

(c) Echelon 4

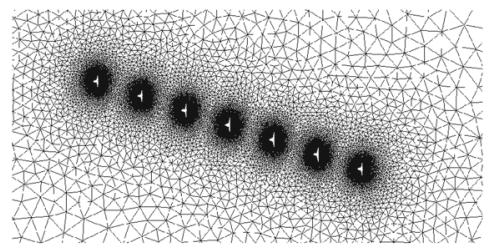

(f) Echelon 7

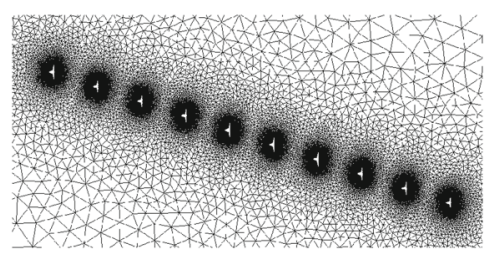

(i) Echelon 10

Fig. 5 Hybrid grids for the echelon formation

\subsection{Proximity Analysis}

As two dimensions are considered proximity between aircraft is accounted for displacement in the $\mathrm{x}$ and $\mathrm{y}$ axis. The y proximity is set at wing tip-to-tip distance of zero. Tip-to-tip distance can be negative as well, where the wingtip of the following aircraft is positioned further inside on the leader's aircraft's wake. It has been reported in the literature that a small tip-to-tip distance will produces the greatest drag reduction for both extended and tight formations [7, 20].

For x-proximity a sensitivity analysis is performed with two aircraft in order to establish the stream-wise distance of aircraft. Four distances are assessed: 1, 2, 3 and 4 wing-spans, Fig. 8 shows the four configurations and and the vortical structure downstream with streamlines. 1
Fig. 6 Hybrid grids for V-type formation

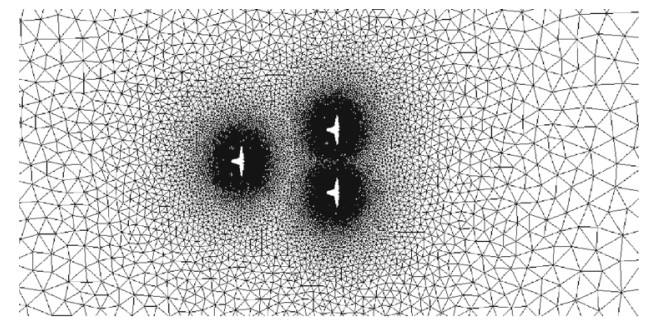

(a) V-type 3

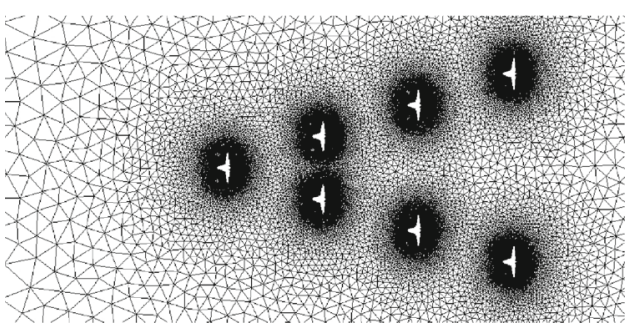

(c) V-type 7

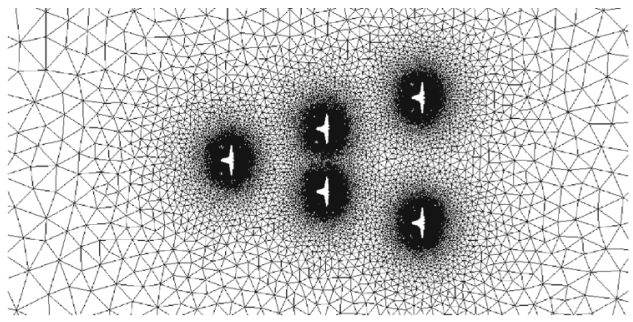

(b) V-type 5

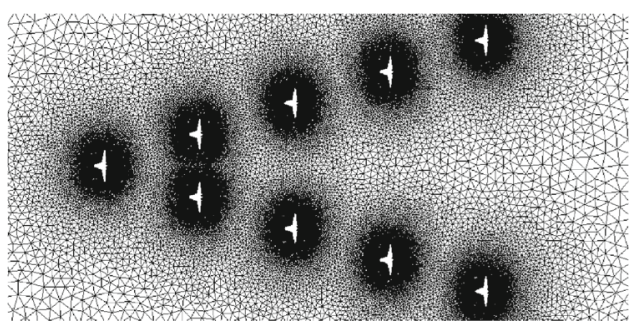

(d) V-type 9 
Fig. 7 Hybrid grids for diamond and half diamond shape formations

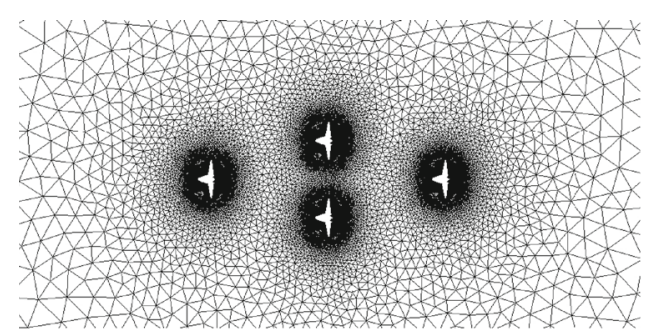

(a) Diamond 4

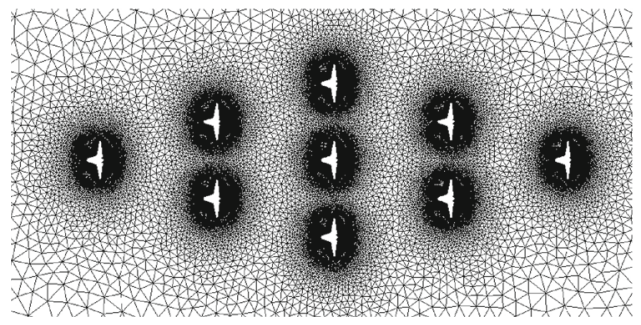

(c) Diamond 9

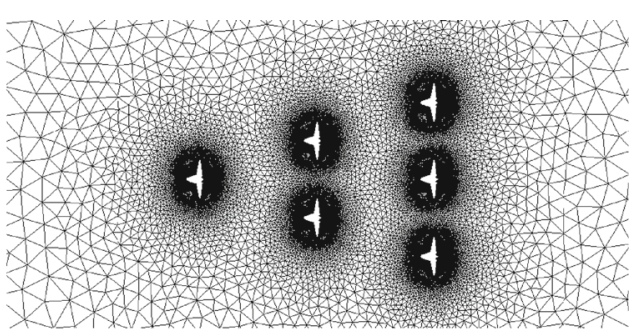

(b) Half-Diamond 6

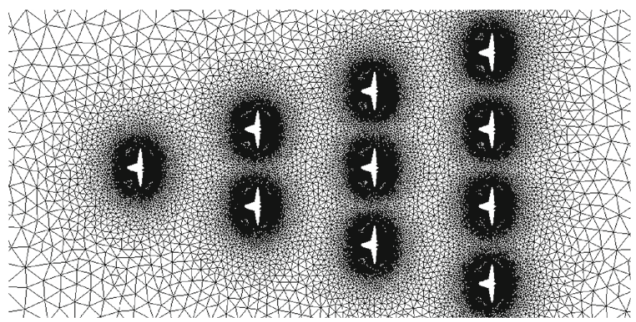

(d) Half-Diamond 10 wing-spans will produce the least drag reduction followed by the 2 with 13.78 and $23.32 \%$ respectively. Increasing the distance to 3 wing-spans further increases the drag reduction by $26.45 \%$ and with 4 wing-spans to $27.21 \%$. The greatest difference is estimated to be between case 1 and 2 , where further increasing the $\mathrm{x}$-distance seems to have an asymptotic trend. In addition, three aircraft simulation on $\mathrm{v}$-type formation are performed with a uniform streamwise distance of 1 and 3 wing-spans. The drag reduction with the greatest distance ( 3 wing-spans) showed an improvement of drag reduction of 1.7 times the improvement with the 1 wing-span proximity. Therefore, a uniform three wingspans streamwise proximity is selected to carry out the complete test-case matrix which is also in-line with tight formation requirement.

\subsection{Simulation Matrix}

A total of 18 test-cases are simulated, where for consistancy a uniform streamwise and tip-to-tip distance is maintained

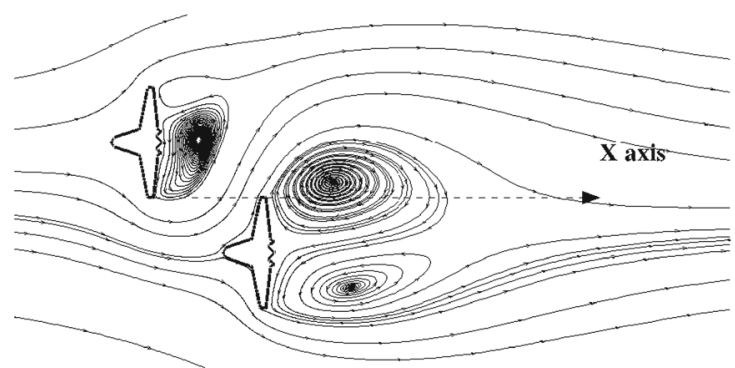

(a) 1 wing-span

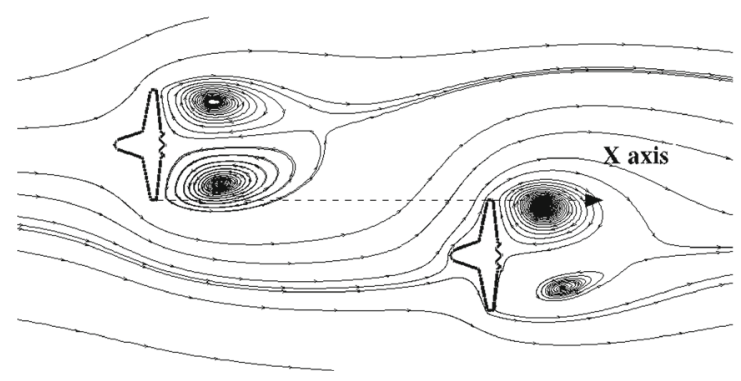

(c) 3 wing-span

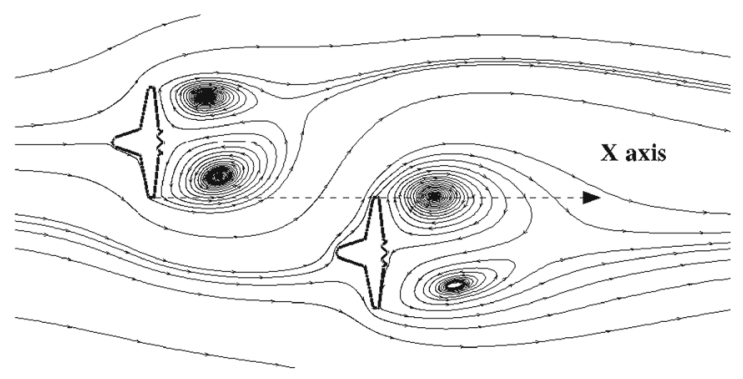

(b) 2 wing-span

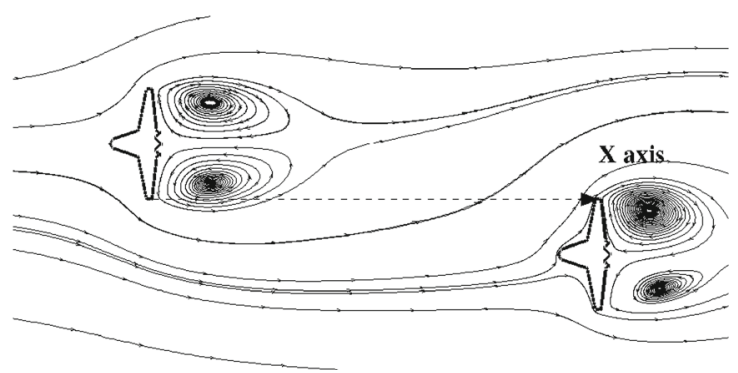

(d) 4 wing-span

Fig. 8 Proximity analysis for $\mathrm{x}$-axis performed with four different wingspans, plotted streamlines for visualising the flow field patterns 
Table 1 Simulation matrix

\begin{tabular}{lllll}
\hline Test-case & Formation shape & Aircraft & Grid elements & Drag reduction (\%) \\
\hline 1 & - & 1 & 29,962 & - \\
2 & Echelon & 2 & 54,146 & $26.45 \%$ \\
3 & Echelon & 3 & 78,538 & $30.96 \%$ \\
4 & Echelon & 4 & 102,864 & $34.21 \%$ \\
5 & Echelon & 5 & 127,062 & $40.86 \%$ \\
6 & Echelon & 6 & 151,350 & $42.93 \%$ \\
7 & Echelon & 7 & 175,530 & $44.08 \%$ \\
8 & Echelon & 8 & 199,820 & $45.25 \%$ \\
9 & Echelon & 9 & 222,798 & $46.02 \%$ \\
10 & Echelon & 10 & 246,918 & $46.89 \%$ \\
11 & V-type & 3 & 96,350 & $34.84 \%$ \\
12 & V-type & 5 & 125,906 & $42.24 \%$ \\
13 & V-type & 7 & 182,544 & $47.84 \%$ \\
14 & V-type & 9 & 276,772 & $51.96 \%$ \\
15 & Diamond & 4 & 101,814 & $48.59 \%$ \\
16 & Half-Diamond & 6 & 148,566 & $47.52 \%$ \\
17 & Diamond & 9 & 220,172 & $65.06 \%$ \\
18 & Half-Diamond & 10 & 242,820 & $57.57 \%$ \\
\hline
\end{tabular}

for all aircraft and formation shapes in detail: 9 echelon, 4 v-type, 2 diamond and 2 half-diamond configurations are simulated. The detailed parameters are summarised in Table 1, containing information for each test-case on the formation shape, number of aircraft, number of grid elements and drag reduction percentages.

\section{Results and Discussion}

This section presents the results from the CFD simulations, quantitatively in terms of drag reduction percentages as well as qualitatively with the use contours plots of Mach number and eddy viscosity.

The benefits of formation flight depended upon the formation shape and number of aircraft is assessed with the total drag for each test-case divided by the number of aircraft in formation and compared with the drag of one aircraft. As expected, increasing the number of aircraft reduces the drag for all formation shapes. It has to be noted that the test-cases with diamond formation are differentiated from full diamond and half diamond illustrated in Fig. 7. Figure 9 presents the drag reduction for all the test-cases, it can be depicted that the most efficient formation is the diamond reaching up to $65 \%$ drag reduction with 9 aircraft where the V-type reaches $52 \%$ and echelon $46 \%$ for the same aircraft number.

The echelon formation is the least efficient, which can be attributed to its sparse configuration. In the other hand, tighter formations such as diamond and half-diamond have better performance. Interestingly enough, the echelon seems to reach an asymptotic behaviour after a sudden drop from 4 to 5 aircraft. The v-type formation commonly encountered in nature (geese and large birds) is more efficient than the echelon, but it doesn't reach the performance of the diamond. The challenge with diamond formation is that it can accommodate specific number of aircraft due to its geometry constraints, the echelon and potentially the vtype with a non-symmetric configuration can accommodate any aircraft number. Moreover, in complicated formation geometry such as diamond, it could require a sophisticated

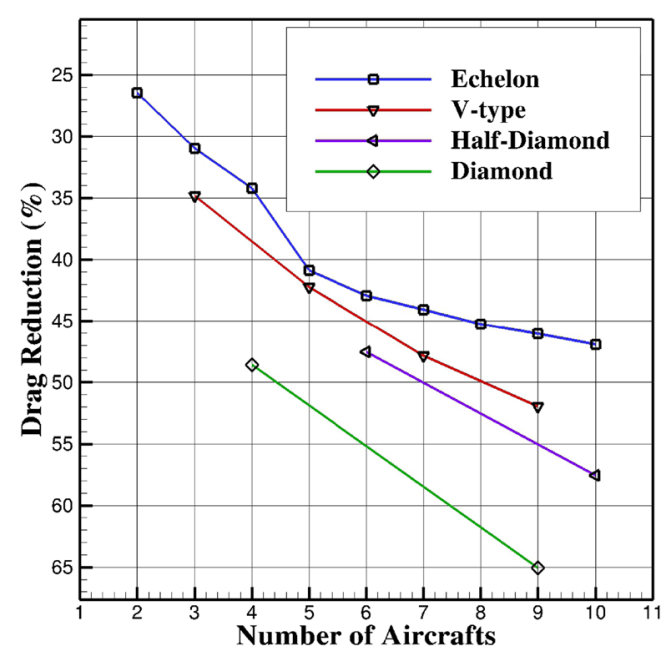

Fig. 9 Drag reduction for the echelon, v-type and diamond formation configurations 


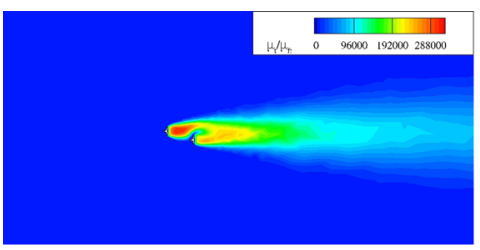

(a) Echelon 2

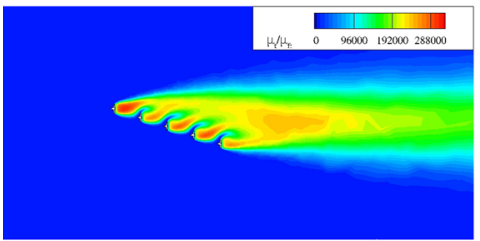

(d) Echelon 5

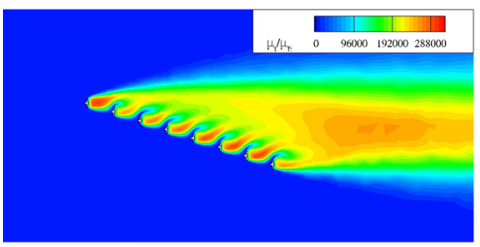

(g) Echelon 8

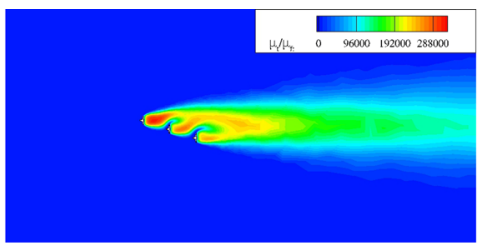

(b) Echelon 3

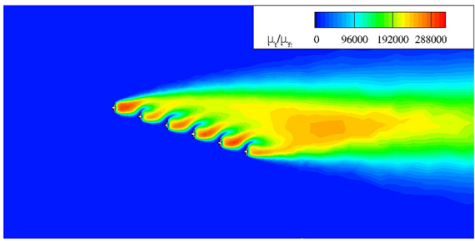

(e) Echelon 6

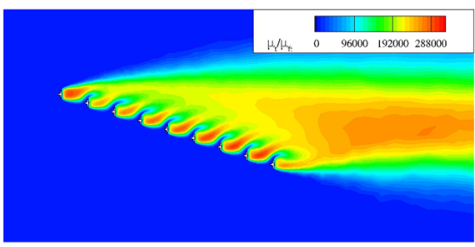

(h) Echelon 9

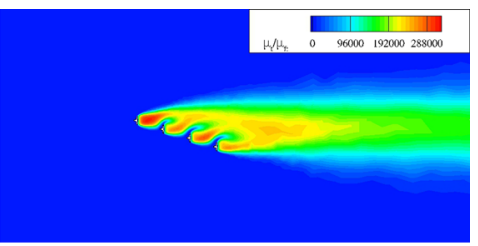

(c) Echelon 4

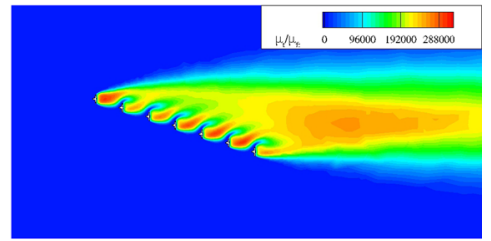

(f) Echelon 7

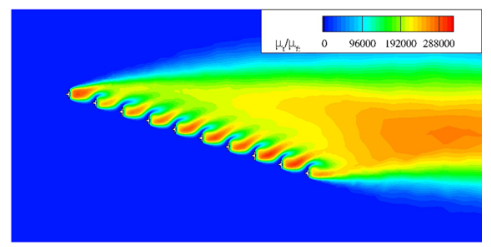

(i) Echelon 10

Fig. 10 Ratio of turbulent viscosity to freestream viscosity for the echelon configuration

rule to split the formation and/or to reshape the formation when encountering a situation.

Even if this analysis is 2D at specific flight conditions similar drag reductions are reported from other studies,
NASA report [3] presents a $20 \%$ with two aircraft at tight formation where the current analysis estimated a $26 \%$. Ning et al. [6] predicted $30 \%$ for two aircraft and $40 \%$ with three, similar reduction is observed with this study, where three

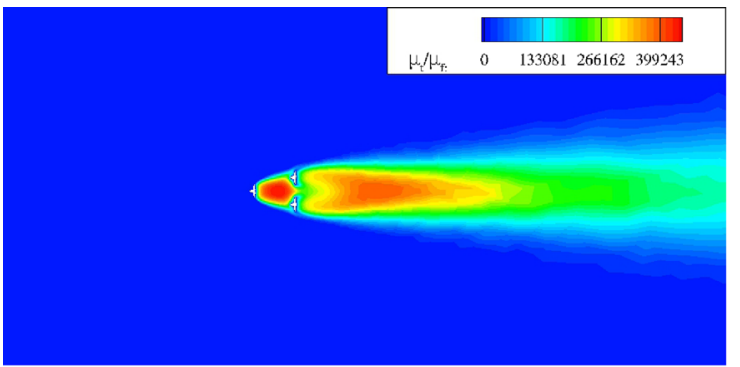

(a) V-type 3

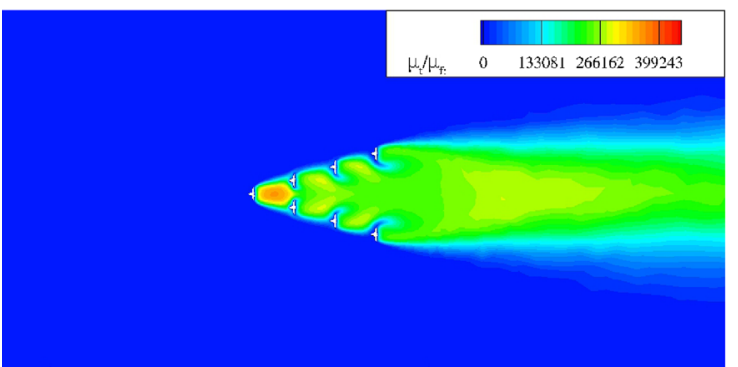

(c) V-type 7

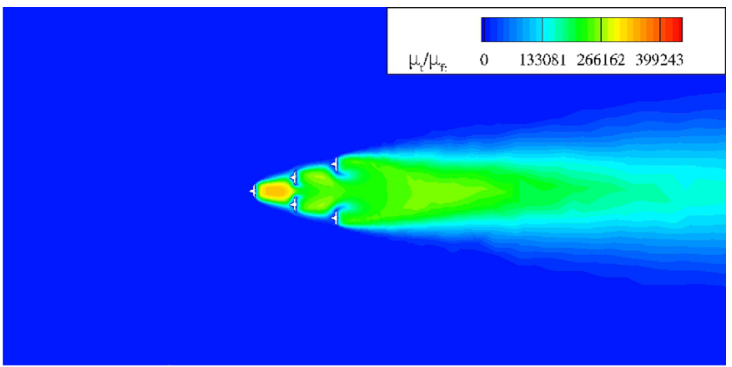

(b) V-type 5

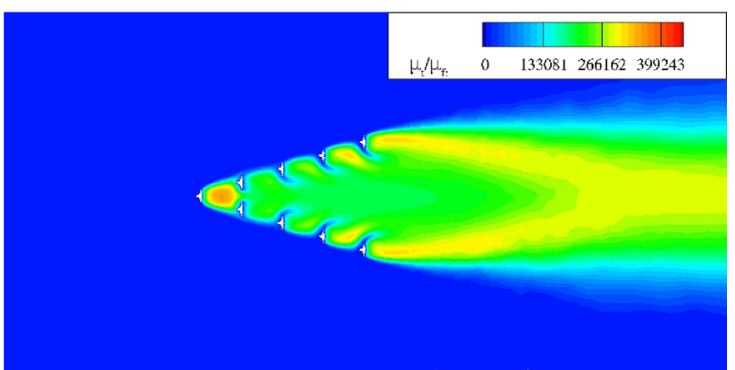

(d) V-type 9

Fig. 11 Ratio of turbulent viscosity to freestream viscosity for the V type formation 


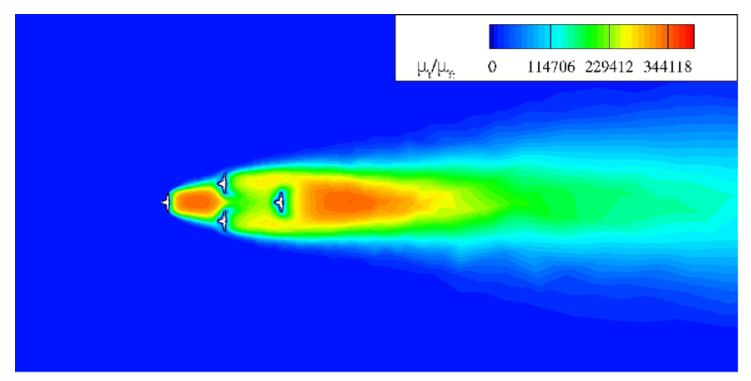

(a) Diamond 4

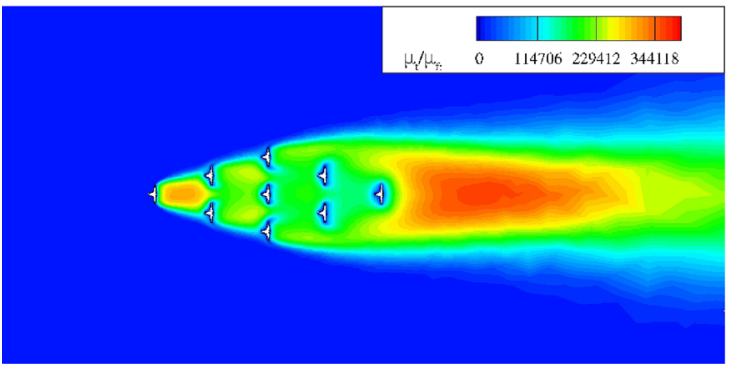

(c) Diamond 9

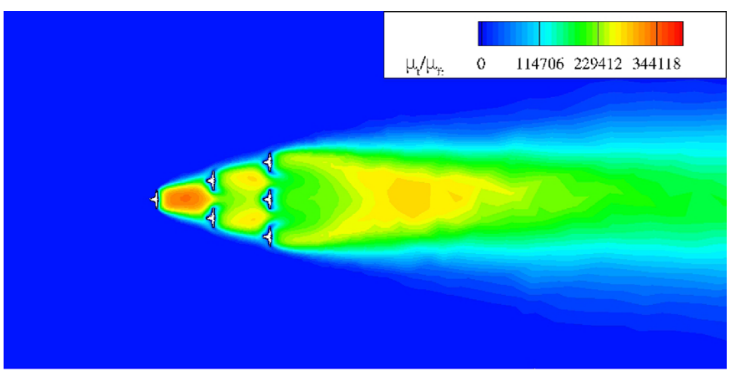

(b) Half-Diamond 6

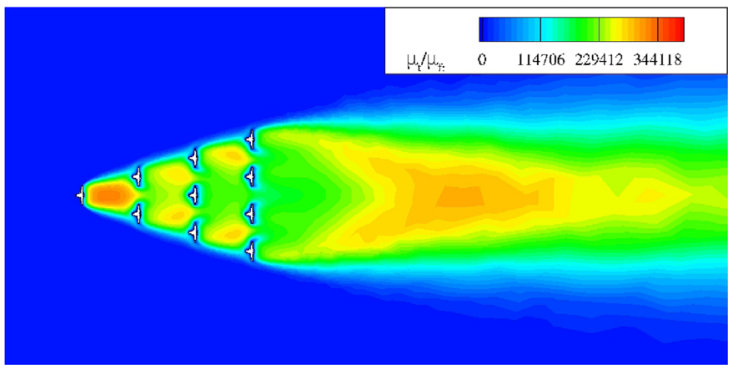

(d) Half-Diamond 10

Fig. 12 Ratio of turbulent viscosity to freestream viscosity for the diamond and half diamond formation

aircraft (V-type) have a $35 \%$ with a reduction difference of $8.5 \%$ compared with $10 \%$ going from two to three aircraft.

The reduction of drag can be correlated also qualitatively with the amount of eddy viscosity computed by the turbulence model. Higher eddy viscosity values in the wake, correspond usually to a more turbulent flow which in terms can have an effect on the computed drag. Figures 10, 11, 12 show the ratio of turbulent viscosity to free-stream viscosity $\left(\mu_{t} / \mu_{\infty}\right)$ for the echelon, V-type and diamond formation respectively. The figures shows that the echelon has a much

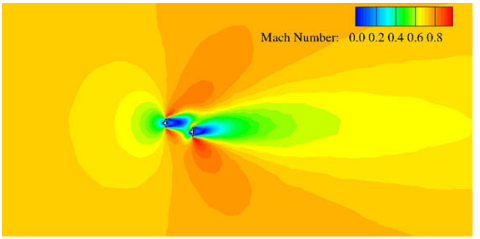

(a) Echelon 2

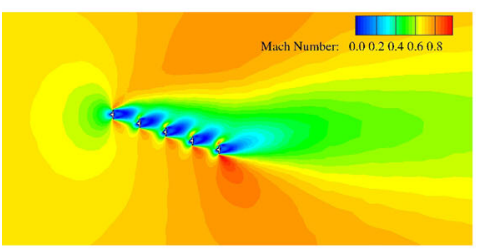

(d) Echelon 5

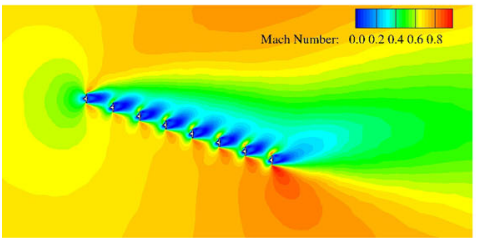

(g) Echelon 8

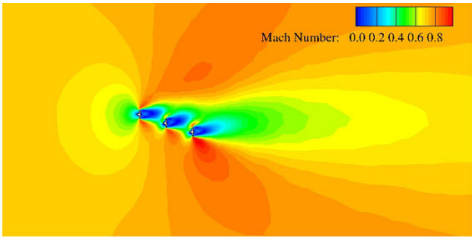

(b) Echelon 3

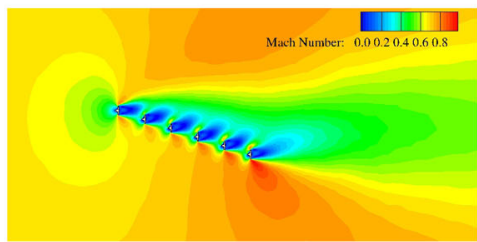

(e) Echelon 6

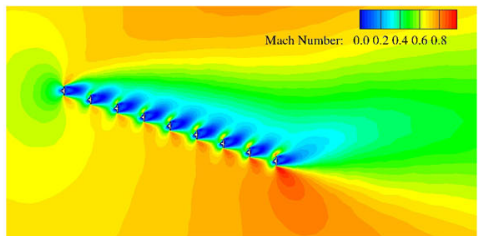

(h) Echelon 9

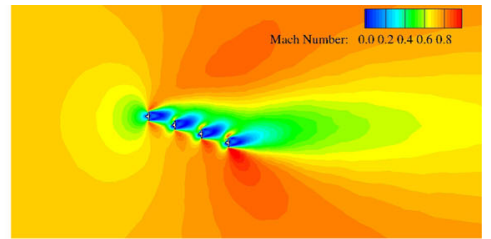

(c) Echelon 4

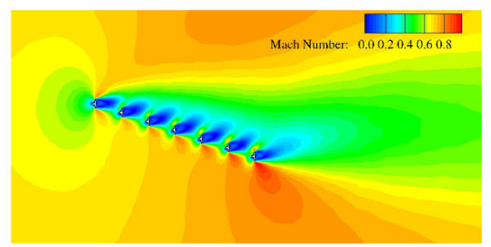

(f) Echelon 7

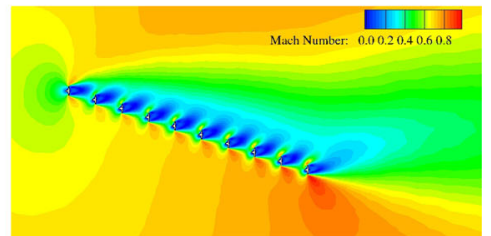

(i) Echelon 10

Fig. 13 Mach number contours for the echelon configuration 


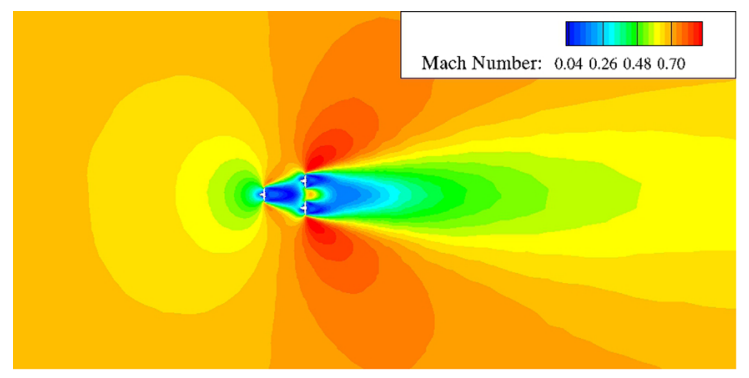

(a) V-type 3

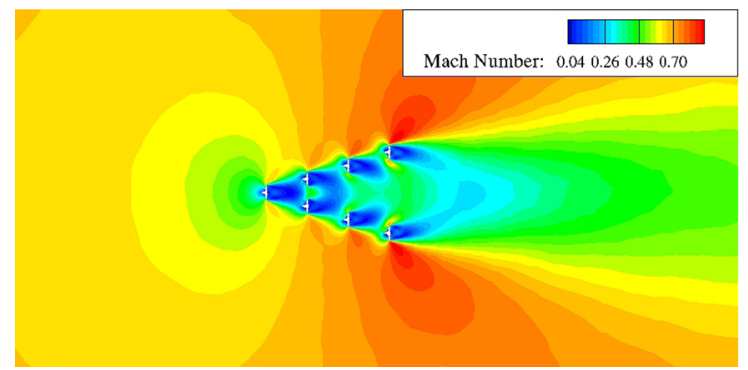

(c) V-type 7

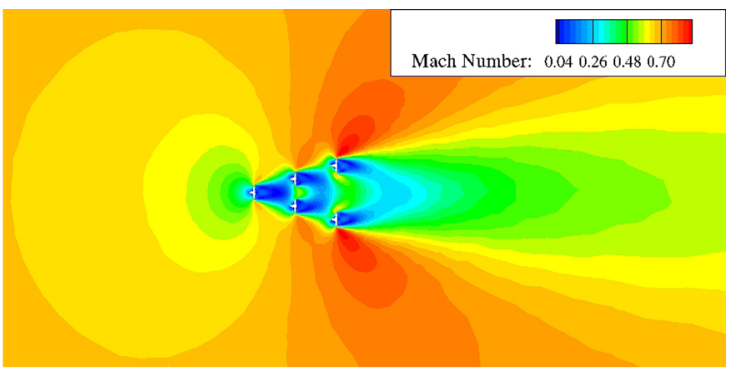

(b) V-type 5

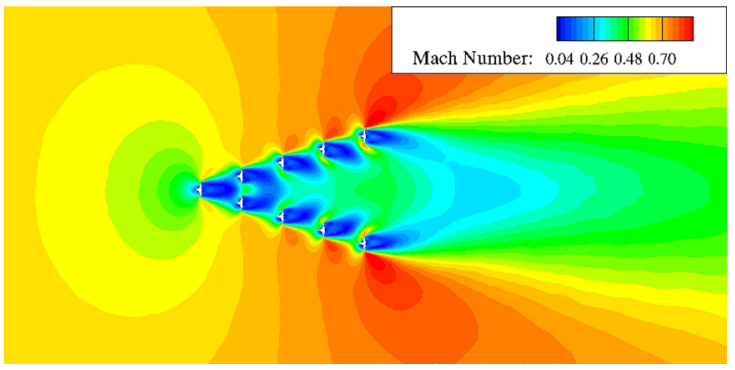

(d) V-type 9

Fig. 14 Mach number contours for the $\mathrm{V}$ type formation

wider wake but lower values of $\mu_{t} / \mu_{\infty}$ compared with the V-type and diamond. The full diamond has higher values of $\mu_{t} / \mu_{\infty}$ than the half diamond and the wake is closer to the trailing aircraft. There are several non-linear effects that they can be observed, for instance the jet-like structure of $\mu_{t} / \mu_{\infty}$ on the V-type 9 test-case downstream the last pair of aircraft, which is not present in the V-5 or V-7. Mach number contours are shown in Figs. 13, 14 and 15. It can be observed that the following aircraft with smaller Mach numbers in their vicinity tend to have a greater drag reduction.

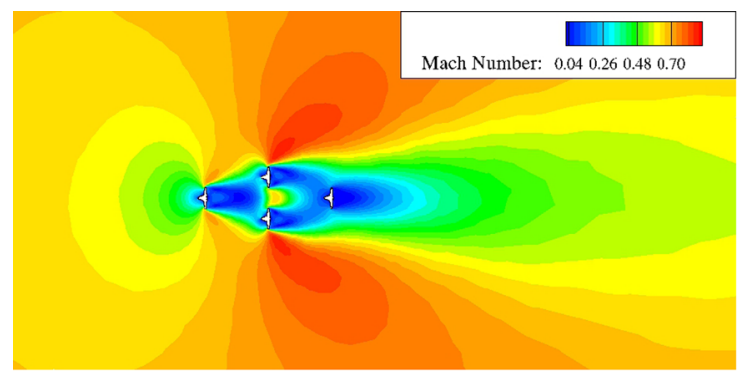

(a) Diamond 4

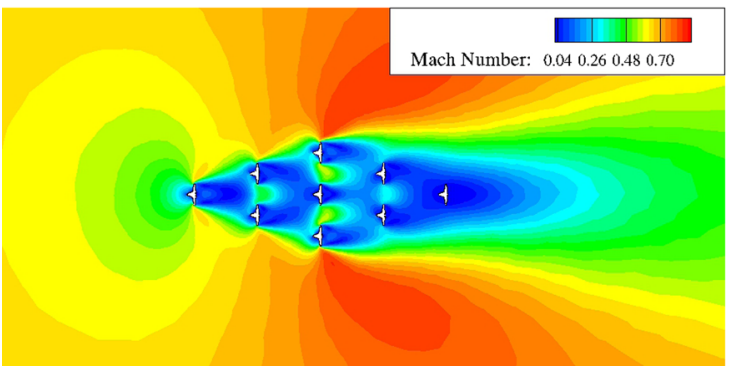

(c) Diamond 9

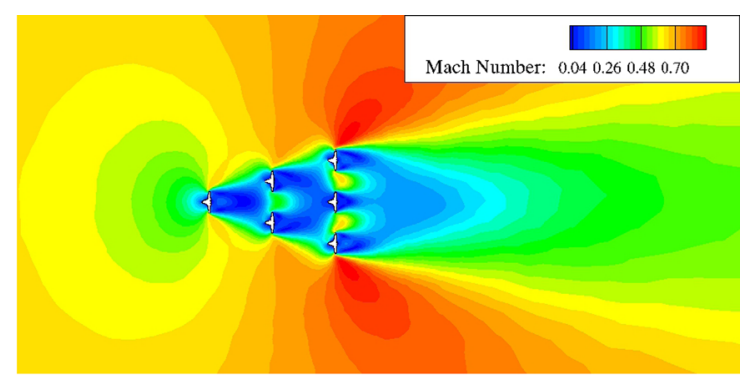

(b) Half-Diamond 6

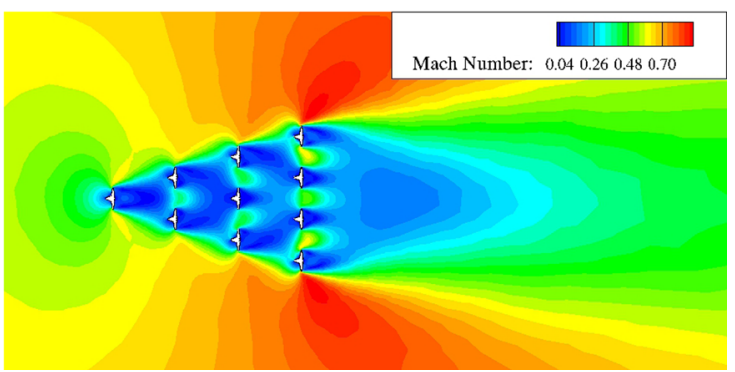

(d) Half-Diamond 10

Fig. 15 Mach number contours for the diamond and half diamond formation 


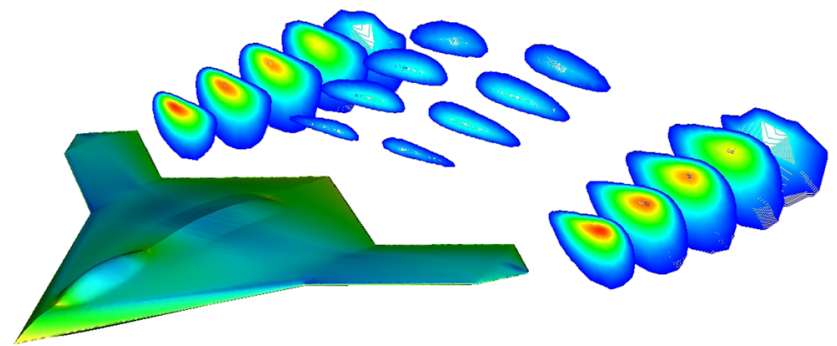

Fig. 16 Surface pressure distribution and contour lines of w velocity

\section{Conclusions and Future work}

This paper comprises 2D CFD computations for UAV flight formation at transonic speeds. Echelon, V-type, diamond and half-diamond formation shape are assessed by also varying the number of aircraft (2 - 10). To establish streamwise proximity distance, an analysis is conducted where three wing-span distance was selected for uniform streamwise spacing. A total of 17 configurations are simulated where drag reduction percentage have been estimated. From the study it was found that the most efficient formation is the diamond type, followed by the half-diamond then the v-type and final the echelon. The predicted drag reductions for two and three aircraft are similar with drag reduction estimates of reported studies.

A 3D inviscid simulation was performed employing the grid shown in Fig. 1, at a Mach number $M_{\infty}=0.7$ and an angle of attack of 1.5. Initial results are shown in Fig. 16 where contours of the pressure distribution are plotted on the aircraft surface and $w$ velocity is used as a mean of identify the wing tip vortex location. More advanced method of identify wing-tip vortices can be employ i.e. q-criterion, $\lambda 2$ and helicity. Furthermore a prismatic 3D grid will enable boundary layer prediction by capturing separation, and transition phenomena. Considering the limitations of the methods used, we might need to prove the benefit of the formation flight and its degree with respect to formation configurations through full realisation. However, this study provides sufficient confidence on the aerodynamic benefit of the formation flying and how it varies depending on the configuration parameters.

Future routes are currently pursuit in order to have a more realistic estimation of the flow in terms of physics and forces prediction. The work in progress includes a multi-fidelity approach to tackled the aerodynamic simulation; the multifidelity framework includes a 3D Vortex Lattice Method coupled with a 3D RANS solver that is able to provide the flexibility, robustness and low computational cost for studying large formation flights. Furthermore several multiobjective optimisation algorithms are employed to estimate the optimal solutions thus optimal position and individual aircraft attitude.

Acknowledgements This work was fully supported by BAE Systems (Operation), who have reviewed and agreed the contents of this paper.

Open Access This article is distributed under the terms of the Creative Commons Attribution 4.0 International License (http:// creativecommons.org/licenses/by/4.0/), which permits unrestricted use, distribution, and reproduction in any medium, provided you give appropriate credit to the original author(s) and the source, provide a link to the Creative Commons license, and indicate if changes were made.

Publisher's Note Springer Nature remains neutral with regard to jurisdictional claims in published maps and institutional affiliations.

\section{References}

1. Weimerskirch, H., Martin, J., Clerquin, Y., Alexandre, P., Jiraskova, S.: Energy saving in flight formation. Nature 413(6857), 697-698 (2001)

2. Bajec, I.L., Heppner, F.H.: Organized flight in birds. Anim. Behav. 78(04), 777-789 (2009)

3. Vachon, M.J., Ray, K.R., Walsh, R.J., Ennix, K.: F/A-18 performance benefits measured during the autonomous formation flight project. Technical Report NASA/TM-2003-210734, National Aeronautics and Space Administration (2003)

4. Garmann, D.J., Visbal, M.R.: Interactions of a streamwiseoriented vortex with a finite wing. J. Fluid Mech. 767, 782-810 (2015)

5. Kless, J.E., Aftosmis, M.J., Ning, S.A., Nemec, M.: Inviscid analysis of extended-formation flight. AIAA J. 51(7), 1703-1715 (2013)

6. Ning, A., Flanzer, T.C., Kroo, I.M.: Aerodynamic performance of extended formation flight. J. Aircr. 48(3), 855-865 (2011)

7. Kaden, A., Luckner, R.: Modeling wake vortex roll-up and vortex-induced forces and moments for tight formation flight. In: AIAA (ed.) AIAA Modeling and Simulation Technologies (MST) Conference (2013)

8. Gunasekaran, M., Mukherjee, R.: Behaviour of trailing wing (s) in echelon formation due to wing twist and aspect ratio. Aerosp. Sci. Technol. 63, 294-303 (2017)

9. Thien, H.P., Moelyadi, M.A., Muhammad, H.: Effects of leaders position and shape on aerodynamic performances of $\mathrm{v}$ flight formation. arXiv:0804.3879 (2008)

10. Shin, H.S., Antoniadis, A.F., Tsourdos, A.: Parametric study on efficient formation flying for a blended-wing uav. In: 2017 International Conference on Unmanned Aircraft Systems (ICUAS), pp. 1657-1664 (2017)

11. Gerz, T., Ehret, T.: Wingtip vortices and exhaust jets during the jet regime of aircraft wakes. Aerosp. Sci. Technol. 1(7), 463-474 (1997)

12. Misaka, T., Holzapfel, F., Gerz, T.: Wake evolution of high-lift configuration from roll-up to vortex decay. In: AIAA (ed.) 51st AIAA Aerospace Sciences Meeting including the New Horizons Forum and Aerospace Exposition 2013 (2013)

13. Spalart, P.R., Allmaras, S.R.: A one-equation turbulence model for aerodynamic flows. AIAA Paper 92-0439, 30th AIAA Aerospace Sciences Meeting (1992) 
14. Antoniadis, A.F., Tsoutsanis, P., Drikakis, D.: High-order schemes on mixed-element unstructured grids for aerodynamic flows. In: AIAA (ed.) 42nd AIAA Fluid Dynamics Conference and Exhibit, number AIAA 2012-2833. New Orleans, Louisiana (2007)

15. Tsoutsanis, P., Titarev, V.A., Drikakis, D.: WENO schemes on arbitrary mixed-element unstructured meshes in three space dimensions. J. Comput. Phys. 230(4), 1585-1601 (2011)

16. Titarev, V.A., Tsoutsanis, P., Drikakis, D.: WENO schemes for mixed-element unstructured meshes. Communications in Computational Physics 8(3), 585-609 (2010)

17. Tsoutsanis, P., Antoniadis, A.F., Drikakis, D.: WENO schemes on arbitrary unstructured meshes for laminar, transitional and turbulent flows. J. Comput. Phys. 256, 254-276 (2014)

18. Rusanov, V.V.: Calculation of interaction of non-steady shock waves with obstacles. J. Comput. Math. Phys. 1(USSR), 267-279 (1961)

19. Luo, H., Baum, J.D., Löhner, R.: A fast, matrix-free implicit method for compressible flows on unstructured grids. J. Comput. Phys. 146(2), 664-690 (1998)

20. Pahle, J., Berger, D., Venti, M., Duggan, C., Faber, J., Cardinal, K.: An initial flight investigation of formation flight for drag reduction on the c-17 aircraft. In: AIAA (ed.) AIAA Atmospheric Flight Mechanics Conference 2012 (2012)

Hyo-Sang Shin received his BSc from Pusan National University in 2004 and gained an MSc on flight dynamics, guidance and control in Aerospace Engineering from KAIST and a PhD on cooperative missile guidance from Cranfield University in 2006 and 2011, respectively. He is currently Reader on Guidance, Control and Navigation Systems in Autonomous and Intelligent Systems Group at Cranfield University. His current research interests include cooperative control of multiple agent systems and multiple target tracking.
Antonis F. Antoniadis is a Lecturer in Computational Engineering Sciences and Director of the Computational Fluid Dynamics (CFD) MSc course at the School of Aerospace Transport and Manufacturing at Cranfield University. Dr Antoniadis has more than 10 years of experience in Computational Fluid Dynamics (CFD) and computer aided engineering methods. He has worked in numerous industrial and government funded projects in the fields of helicopter aerodynamics, unmanned aerial vehicles, morphing technologies, supersonic flows, combustion, virtualisation and visualisation, high performance computing amongst others. Clients include JLR, BAEsystems, AIRBUS, Red-Bull technologies, Qinetiq, EPSRC, and European Commission.

Antonios Tsourdos obtained a MEng in electronic, control and systems engineering from the University of Sheffield (1995), an MSc in systems engineering from Cardiff University (1996), and a $\mathrm{PhD}$ in nonlinear robust missile autopilot design and analysis from Cranfield University (1999). He is a Professor of Control Engineering with Cranfield University, and was appointed Head of the Centre for Autonomous and Cyber-Physical Systems in 2013. He was a member of the Team Stellar, the winning team for the UK MoD Grand Challenge (2008) and the IET Innovation Award (Category Team, 2009). 